\title{
Vertex integrals in heavy-particle theories
}

\author{
Antonio O. Bouzas \\ Departamento de Física Aplicada, Cinvestav-IPN, Apdo. Postal 73 “Cordemex”, Mérida 97310, \\ Yucatán, México
}

\begin{abstract}
We give the results of complete analytical computations of two- and three-point loop integrals ocurring in heavy particle theories, with and without velocity change, for arbitrary values of external momenta and masses.
\end{abstract}

\section{INTRODUCTION}

In this talk we consider a class of one-loop integrals occurring in heavy-particle theories [1], with arbitrary real values for the external masses and residual momenta. We give the results of complete analytical computations of three-point loop integrals with and without velocity change, and two-point loop integrals. The details of the calculations are given in [2], and in a forthcoming paper.

\section{LOOP INTEGRALS}

The loop integrals we consider are of the form,

$$
\begin{aligned}
g_{2}^{\alpha_{1} \cdots \alpha_{n}}= & \frac{i \mu^{4-d}}{(2 \pi)^{d}} \int d^{d} \ell \frac{\ell^{\alpha_{1}} \cdots \ell^{\alpha_{n}}}{(2 v \cdot(\ell+k)-\delta M+i \varepsilon)\left(\ell^{2}-m^{2}+i \varepsilon\right)} . \\
g_{3}^{\alpha_{1} \cdots \alpha_{n}}= & \frac{i \mu^{4-d}}{(2 \pi)^{d}} \int d^{d} \ell \frac{\ell^{\alpha_{1}} \cdots \ell^{\alpha_{n}}}{\left(2 v_{1} \cdot\left(\ell+k_{1}\right)-\delta M_{1}+i \varepsilon\right)\left(2 v_{2} \cdot\left(\ell+k_{2}\right)-\delta M_{2}+i \varepsilon\right)} \\
& \times \frac{1}{\left(\ell^{2}-m^{2}+i \varepsilon\right)} \\
\mathcal{H}_{3}^{\alpha_{1} \cdots \alpha_{n}}= & \frac{i \mu^{4-d}}{(2 \pi)^{d}} \int d^{d} \ell \frac{\ell^{\alpha_{1}} \cdots \ell^{\alpha_{n}}}{(2 v \cdot \ell-\delta M+i \varepsilon)\left(\left(\ell-k_{1}\right)^{2}-m^{2}+i \varepsilon\right)} \\
& \times \frac{1}{\left(\left(\ell-k_{2}\right)^{2}-m^{\prime 2}+i \varepsilon\right)}
\end{aligned}
$$

Here $v_{i}^{\mu}, i=1,2$, are the velocities of the external heavy legs, $k_{i}^{\mu}$ their residual momenta, and $\delta M_{i}$ their mass splittings relative to the common heavy mass of the corresponding heavy quark symmetry multiplet. $m$ and $m^{\prime}$ are the masses of the light particles within the loops, which in chiral theories are light pseudoscalar mesons. These integrals are defined in $d=4-\varepsilon$ dimensions, $\mu$ being the mass scale of dimensional regularization. 
Their degrees of divergence are $n+d-3$ for $g_{2}^{\alpha_{1} \cdots \alpha_{n}}, n+d-4$ for $g_{3}^{\alpha_{1} \cdots \alpha_{n}}$ and $n+d-5$ for $\mathcal{H}_{3}^{\alpha_{1} \cdots \alpha_{n}}$. The factor of 2 in front of $v_{i}^{\mu}$ corresponds to our normalization of the heavyparticle propagators.

Our method of calculation [2] is to obtain the integrals (1) as large-mass limits of ordinary loop integrals. We closely follow the approach of [3] for the computation of scalar integrals, which are greatly simplified in the large-mass limit, and the method of [4] to express tensor integrals in terms of scalar ones.

\section{TWO-POINT INTEGRALS}

Two-point integrals with one heavy propagator have been given in $[2,5,6]$. The scalar two-point integral $g_{2}$ is a function of $m$ and $\Delta=\delta M-2 v \cdot k$. We write it in terms of $\xi=\Delta /(2 m)$,

$$
I_{2}(\Delta, m)=\frac{\Delta}{32 \pi^{2}}\left(\frac{2}{\varepsilon}+\log \left(\frac{\bar{\mu}^{2}}{m^{2}}\right)+2\right)+\frac{m}{16 \pi^{2}} \mathcal{F}(\xi)
$$

with $\mathcal{F}(x)=\sqrt{x^{2}-1+i \varepsilon}\left[\log \left(x-\sqrt{x^{2}-1+i \varepsilon}\right)-\log \left(x+\sqrt{x^{2}-1+i \varepsilon}\right)\right]$. The coefficient of the dimensional regularization pole vanishes when $\Delta=0$. This is due to the fact that the real part of the integrand in $g_{2}$ is parity-odd when $\Delta=0$.

The vector two-point integral $g_{2}^{\mu}\left(v^{\alpha}, \Delta, m\right)$ is given in terms of only one form factor, $g_{2}^{\mu}\left(v^{\alpha}, \Delta, m\right)=F(\Delta, m) v^{\mu}$, with $F(\Delta, m)=v_{\mu} g_{2}^{\mu}\left(v^{\alpha}, \Delta, m\right)=1 / 2 A_{0}(m)+\Delta / 2 g_{2}(\Delta, m)$, where $A_{0}$ is the standard one-point scalar integral (see the appendix of [2]). The secondrank tensor integral is computed analogously, explicit results being given in [2].

\section{THREE-POINT INTEGRALS WITH VELOCITY CHANGE}

The scalar three-point integral with velocity change, $g_{3}=I_{3}\left(v_{1} \cdot v_{2}, \Delta_{1}, \Delta_{2}, m\right)$, where $\Delta_{j}=\delta M_{j}-2 v_{j} \cdot k_{j}$, can be expressed in terms of four dilogarithms [2],

$$
g_{3}=\frac{1}{64 \pi^{2}} \frac{1-\Omega^{2}}{\Omega}\left\{\frac{2}{\varepsilon} \log (\alpha)+\log ^{2}(\alpha)+\sum_{k, \sigma}(-1)^{k+1}\left[\frac{1}{2} \log ^{2}\left(\frac{-z_{k \sigma}}{\bar{\mu}}\right)+\operatorname{Li}_{2}\left(\frac{-y_{0}}{z_{k \sigma}}\right)\right]\right\} .
$$

The notation is as follows, $\omega=v_{1} \cdot v_{2}, \Omega=\sqrt{(\omega-1) /(\omega+1)}, \alpha=\omega+\sqrt{\omega^{2}-1}=$ $(1+\Omega) /(1-\Omega)$ and $\bar{\mu}$ is the mass unit in the $\overline{\mathrm{MS}}$ scheme. The sum extends over $k=1,2$ and $\sigma= \pm$, with

$$
\begin{aligned}
y_{0} & =-\frac{1+\Omega}{2 \Omega}\left(\frac{1+\Omega}{2} \Delta_{1}-\frac{1-\Omega}{2} \Delta_{2}\right) \\
z_{1 \pm} & =\frac{1}{2}\left(\frac{1+\Omega^{2}}{2 \Omega} \Delta_{1}-\frac{1-\Omega^{2}}{2 \Omega} \Delta_{2} \pm \sqrt{\Delta_{1}^{2}-4 m^{2}+i \varepsilon}\right) \\
z_{2 \pm} & =\frac{\alpha}{2}\left(\frac{1-\Omega^{2}}{2 \Omega} \Delta_{1}-\frac{1+\Omega^{2}}{2 \Omega} \Delta_{2} \pm \sqrt{\Delta_{2}^{2}-4 m^{2}+i \varepsilon}\right) .
\end{aligned}
$$


This expression for $\mathcal{J}_{3}$ is equivalent to the result given in eq. (30) of [2], though it has been written in a more compact form by means of the identity

$$
\frac{1}{2} \log ^{2}(z)-\log (z) \log (-z)=-\frac{\pi^{2}}{2}-\frac{1}{2} \log ^{2}(-z)
$$

valid on the first Riemann sheet of the logarithm, and the identity (A.2) of [3] for the dilogarithm.

In order to compute the vector integral $g_{3}^{\mu}$ we define two sets of form factors as $g_{3}^{\mu}=I_{1} v_{1}^{\mu}+I_{2} v_{2}^{\mu}$ and $F_{1,2}=v_{1,2} \cdot g_{3}$. These form factors are given by,

$I_{1}=\frac{1-\Omega^{2}}{4 \Omega^{2}}\left[-\left(1-\Omega^{2}\right) F_{1}+\left(1+\Omega^{2}\right) F_{2}\right], \quad I_{2}=\frac{1-\Omega^{2}}{4 \Omega^{2}}\left[\left(1+\Omega^{2}\right) F_{1}-\left(1-\Omega^{2}\right) F_{2}\right]$,

with $F_{1,2}=1 / 2 g_{2}\left(\Delta_{2,1}, m\right)+\Delta_{1,2} / 2 J_{3}\left(v_{1} \cdot v_{2}, \Delta_{1}, \Delta_{2}, m\right)$. These equations give an explicit expression for $g_{3}^{\mu}$. For the sake of brevity, we omit here the results for the tensor integral $g_{3}^{\mu \nu}$, which can be found in [2].

\section{THREE-POINT INTEGRALS WITH ONE HEAVY PROPAGATOR}

The scalar three-point integral $\mathcal{H}_{3}=\mathcal{H}_{3}\left(v \cdot q, q^{2}, \Delta, m, m^{\prime}\right)$, with $q=\left(k_{2}-k_{1}\right) / 2$ and $\Delta=\delta M-v \cdot\left(k_{1}+k_{2}\right)$, can be expressed in terms of eight dilogarithms as,

$$
\begin{aligned}
\mathcal{H}_{3} & =\frac{1}{(4 \pi)^{2}} \frac{1}{4|\mathbf{q}|} \sum_{j=1,2}\left(\mathcal{F}_{1}\left(y_{j}\right)+\mathcal{F}_{2}\left(x_{j}\right)-\mathcal{F}_{3}\left(z_{j}\right)\right) \\
\mathcal{F}_{1}(x) & =\operatorname{Li}_{2}\left(\frac{z_{0}-4|\mathbf{q}| \alpha}{z_{0}-4|\mathbf{q}| x}\right)-\operatorname{Li}_{2}\left(\frac{z_{0}}{z_{0}-4|\mathbf{q}| x}\right) \\
\mathcal{F}_{2}(x) & =-\operatorname{Li}_{2}\left(\frac{z_{0}-4|\mathbf{q}| \alpha}{z_{0}-4|\mathbf{q}| x}\right)-\frac{1}{2} \log ^{2}\left(\frac{z_{0}-4|\mathbf{q}| x}{\mu^{2}}\right) \\
\mathcal{F}_{3}(x) & \equiv \mathcal{F}_{1}(x)+\mathcal{F}_{2}(x)=-\operatorname{Li}_{2}\left(\frac{z_{0}}{z_{0}-4|\mathbf{q}| x}\right)-\frac{1}{2} \log ^{2}\left(\frac{z_{0}-4|\mathbf{q}| x}{\mu^{2}}\right)
\end{aligned}
$$

The quantities entering these equations are $|\mathbf{q}|=\sqrt{(v \cdot q)^{2}-q^{2}}(|\mathbf{q}|$ is assumed to be real $), \alpha \equiv \alpha_{+}$with $\alpha_{ \pm}=2(v \cdot q \pm|\mathbf{q}|), z_{0}=-\left(m^{\prime 2}-m^{2}\right)-\alpha_{+}(\Delta-2|\mathbf{q}|)$, and,

$$
\begin{aligned}
x_{1,2} & =v \cdot k_{2}+2|\mathbf{q}|-\frac{\delta M}{2} \pm \sqrt{\left(v \cdot k_{1}-\frac{\delta M}{2}\right)^{2}-m^{2}+i \varepsilon} \\
y_{1,2} & =\frac{1}{2 \alpha_{-}}\left(4 q^{2}+m^{\prime 2}-m^{2} \pm \sqrt{\left(4 q^{2}-\left(m^{\prime}+m\right)^{2}\right)\left(4 q^{2}-\left(m^{\prime}-m\right)^{2}\right)+i \varepsilon \sigma}\right) \\
z_{1,2} & =v \cdot k_{2}-\frac{\delta M}{2} \pm \sqrt{\left(v \cdot k_{2}-\frac{\delta M}{2}\right)^{2}-m^{\prime 2}+i \varepsilon}
\end{aligned}
$$


where in the expression for $y_{j}$ we denoted $\sigma \equiv \operatorname{sgn}\left(q^{2}\right) . \mu$ is a positive constant with dimension of mass, analogous to the mass unit in dimensional regularization. It is not difficult to show that $\mathcal{H}_{3}$ does not depend on $\mu$, it appears there for purely dimensional reasons.

Tensor three-point integrals $\mathcal{H}_{3}^{\alpha_{1} \cdots \alpha_{n}}=\mathcal{H}_{3}^{\alpha_{1} \cdots \alpha_{n}}\left(v, k_{1}, k_{2} ; \delta M, m, m^{\prime}\right)$ can be given in terms of integrals with smaller ranks and fewer points, by the well-known method of [4]. We will consider integrals of standard form $\mathrm{H}_{3}^{\alpha_{1} \cdots \alpha_{n}}\left(v, q ; \Delta, m, m^{\prime}\right)=$ $\mathcal{H}_{3}^{\alpha_{1} \cdots \alpha_{n}}\left(v,-q, q ; \Delta+v \cdot\left(k_{1}+k_{2}\right), m, m^{\prime}\right)$ in terms of which we can express $\mathcal{H}_{3}$ as,

$$
\mathcal{H}_{3}^{\alpha_{1} \cdots \alpha_{n}}=\mathrm{H}_{3}^{\alpha_{1} \cdots \alpha_{n}}\left(v, q ; \Delta, m, m^{\prime}\right)+\sum_{j=1}^{n} r^{\left\{\alpha_{1}\right.} \cdots r^{\alpha_{j}} \mathrm{H}_{3}^{\left.\alpha_{j+1} \cdots \alpha_{n}\right\}}\left(v, q ; \Delta, m, m^{\prime}\right),
$$

where $r^{\mu} \equiv 1 / 2\left(p^{\prime}+p\right)^{\mu}$, and $A^{\left\{\alpha_{1} \alpha_{2} \cdots \alpha_{s}\right\}} \equiv A^{\alpha_{1} \alpha_{2} \cdots \alpha_{s}}+A^{\alpha_{2} \cdots \alpha_{s} \alpha_{1}}+\cdots+A^{\alpha_{s} \alpha_{1} \cdots \alpha_{s-1}}$. Clearly, for the scalar integral we have $\mathcal{H}_{3}=\mathrm{H}_{3}$.

For the vector integral we write $\mathrm{H}_{3}^{\alpha}\left(v, q ; \Delta, m_{1}, m_{2}\right)=\mathrm{V} v^{\alpha}+\mathrm{Q} q^{\alpha}$, with $\mathrm{V}=\mathrm{V}(v$. $\left.q, q^{2}, \Delta, m_{1}, m_{2}\right)$ and similarly $\mathrm{Q}$. If $(v \cdot q)^{2}-q^{2}=|\mathbf{q}|^{2}=0$, then $q^{\alpha} \propto v^{\alpha}$ and we can set $\mathrm{Q}=0$. If $|\mathbf{q}|^{2} \neq 0$,

$$
|\mathbf{q}|^{2} \mathrm{Q}=v \cdot q v_{\alpha} \mathrm{H}_{3}^{\alpha}-q_{\alpha} \mathrm{H}_{3}^{\alpha}, \quad|\mathbf{q}|^{2} \mathrm{~V}=-q^{2} v_{\alpha} \mathrm{H}_{3}^{\alpha}-v \cdot q q_{\alpha} \mathrm{H}_{3}^{\alpha},
$$

with,

$$
\begin{aligned}
v_{\alpha} \mathrm{H}_{3}^{\alpha} & =\frac{1}{2} B_{0}\left(4 q^{2}, m_{1}, m_{2}\right)+\frac{\Delta}{2} \mathrm{H}_{3} \\
q_{\alpha} \mathrm{H}_{3}^{\alpha} & =\frac{1}{4} g_{2}\left(\Delta-2 v \cdot q, m_{2}\right)-\frac{1}{4} g_{2}\left(\Delta+2 v \cdot q, m_{1}\right)+\frac{m_{1}^{2}-m_{2}^{2}}{4} \mathrm{H}_{3} .
\end{aligned}
$$

We have omitted the arguments $\left(v, q ; \Delta, m_{1}, m_{2}\right)$ of $\mathrm{H}_{3}^{\alpha}$ on boths sides of these equations for brevity. $B_{0}$ is the standard scalar two-point integral, as given in the appendix of [2]. Higher-rank tensor integrals can be computed analogously.

The results presented in this section were obtained in collaboration with R. Flores Mendieta. A detailed derivation will be given elsewhere.

\section{ACKNOWLEDGEMENTS}

This work has been partially supported by Conacyt and SNI.

\section{REFERENCES}

1. See, e.g., A.Manohar, M.Wise, "Heavy Quark Physics,” Cambridge Univ. Press, 2000, and references therein.

2. A.Bouzas, Eur.Phys.J. C12, (2000), 643

3. G.'t Hooft, M.Veltman, Nucl.Phys. B153, (1979), 365.

4. G.Passarino, M.Veltman, Nucl.Phys. B160, (1979), 151.

5. D.Broadhurst,A.Grozin, Phys.Lett. B267, (1991), 105.

6. I.W.Stewart, Nucl.Phys. B529, (1998), 62. 